\title{
Adult Meningeal Melanoma
}

National Cancer Institute

\section{Source}

National Cancer Institute. Adult Meningeal Melanoma. NCI Thesaurus. Code C5319.

A melanoma that arises from leptomeningeal melanocytes and occurs in adulthood. 